\title{
Aging in dense suspensions of soft thermosensitive microgel particles studied with particle-tracking microrheology
}

\author{
Dirk van den Ende, ${ }^{1}$ Eko H. Purnomo, ${ }^{1}$ Michel H. G. Duits, ${ }^{1}$ Walter Richtering, ${ }^{2}$ and Frieder Mugele ${ }^{1}$ \\ ${ }^{1}$ Physics of Complex Fluids, IMPACT and MESA + Institute, Department of Science and Technology, University of Twente, \\ P.O. Box 217, 7500 AE Enschede, The Netherlands \\ ${ }^{2}$ Institute of Physical Chemistry, RWTH Aachen University, Landoltweg 2, D-52056 Aachen, Germany \\ (Received 24 September 2009; revised manuscript received 9 December 2009; published 13 January 2010)
}

\begin{abstract}
Using particle tracking microrheology, we studied the glass transition in dense suspensions of thermosensitive microgel particles. These suspensions can be tuned reversibly between the glass state at low temperature and the liquid state at high temperature. In the glass state, the ensemble averaged mean squared displacements (MSDs) of added fluorescent tracer particles depend on the age of the suspension. We also determine the local viscoelastic moduli, $G^{\prime}$ and $G^{\prime \prime}$, from the MSDs using the Generalized Stokes-Einstein Relation and compare them to the bulk moduli, measured using conventional rheometry. With particle tracking, one probes the viscoelastic moduli in a lower frequency range than with macrorheology, which makes it possible to determine the mean relaxation time that is inaccessible with macrorheology. In the glass state, the mean relaxation time increases linearly with the age of the sample and the short time particle displacement distributions are nonGaussian, indicating inhomogeneity of the system. The observed difference between conventional and microrheology is explained quantitatively assuming that the tracer particles are surrounded by a viscoelastic liquid shell, different from the bulk.
\end{abstract}

DOI: 10.1103/PhysRevE.81.011404

PACS number(s): 83.80.Hj, 83.60.Bc, 83.80.Kn

\section{INTRODUCTION}

Glass transitions [1], aging [2-6], dynamic heterogeneity [7], and slow relaxation processes [8] are topics of interest in the study of soft glassy materials. These topics are mostly studied using colloidal systems due to their larger size, which inherently provides longer time scales than atomic and molecular systems. Moreover, the chemical and physical properties of colloidal particles can be flexibly manipulated [9].

For a colloidal hard sphere system, a glass transition is normally achieved by increasing its volume fraction via the mass concentration of the hard colloidal particles. As the system approaches the glass transition, its structural length scale (cluster size) increases and this increase is responsible for slowing down the dynamics [1]. This slowing down has been observed with light scattering experiments on colloidal hard sphere dispersions, since a second plateau in the intensity correlation occurs when the glass transition is approached. It is well described by the mode coupling theory [10]. The mode coupling theory has also been applied successfully to describe quantitatively the flow curve of thermosensitive microgel particle suspensions as they approach the glass transition [11] and the viscoelastic moduli of a dense hard-sphere suspension as function of the applied frequency and strain amplitude [12]. However, to account for the influence of aging on the rheological properties, this mode coupling theory is still under development [13]. On the other hand, the phenomenological soft glassy rheology model predicts not only the rheological behavior as the system approaches the glass transition but also deep in the glassy state where the suspension shows aging [14-16].

Although the glass transition in colloidal hard sphere suspensions was widely studied [17-21], the dynamic behavior of soft colloidal systems around the glass transition is hardly investigated. Examples of model soft systems are star poly- mers [22] and polyelectrolyte microgels [23]. In this study, we use thermosensitive core-shell microgel particles, in which the core consists of thermosensitive poly-N-isopropyl acrylamide (polyNipam) and the shell is poly-N-isopropyl methacrylamide (polyNipmam) polymer [24-26]. The size of the particle can be controlled reversibly by tuning the temperature, which provides a unique way to control the volume fraction. Compared to the usual thermosensitive polyNipam system, the size of the core-shell particle varies more gradually with temperature resulting in a wider temperature range to tune the particle size [5].

In previous studies [4-6], we investigated the glass transition of dense suspensions of these soft particles as well as the aging in the glassy state using macrorheology. At high temperatures, the system was liquid like (i.e., the loss modulus was higher than the elastic modulus and the moduli measured at a certain frequency were age-independent). In contrast, at low temperature, when the particles are swollen, the elastic modulus is higher than the loss modulus indicating that the system is solidlike. We also found that both the elastic and the loss modulus depend on the age $t$ of the system; the mean relaxation time $\tau$ of the aging suspension scales with $t$. However, we were not able to measure directly this mean relaxation time. Because the relaxation times scale with the age of the sample, the viscoelastic moduli are a function of $\omega t$ in stead of $\omega$. The lowest $\omega t$ at which the viscoelastic moduli in an oscillatory experiment can be determined, is about $\pi / 2$, hence the longest detectable relaxation time is $\tau_{\max }=2 t / \pi$. To investigate this age dependence of the relaxation times and the microscopic dynamics of a the suspensions near the glass transition, we use in this study particle tracking microrheology by analyzing the motion of probe particles added to the suspension.

Qualitatively, similar behavior is observed as in the macrorheology experiments. At low temperatures the ensemble 


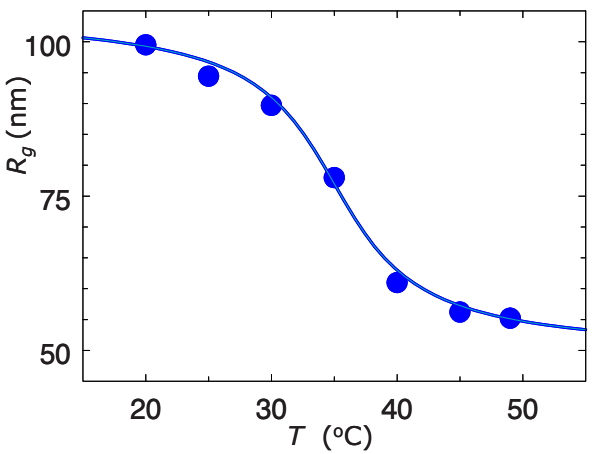

FIG. 1. (Color online) The radius of gyration $R_{g}$ of a polyNipam-polyNipmam microgel particle as a function of the temperature $T$ measured with static light scattering. The line is drawn to guide the eye.

averaged mean squared displacement (MSD) of the probe particles measured over a time interval $t-t_{w}$ (where $t_{w}$ is the age of the sample at the start of the tracking) is at short times independent of the tracking time $t-t_{w}$ and diffusive at long times, i.e., the MSD increases linearly with tracking time. This indicates a caging-escape behavior typical for glassy systems. The caging plateau of the MSD vanishes as we increase the temperature and the MSD becomes diffusive. We also observe aging in the glassy state (low temperature) as shown by the increase in the crossover time from caging to diffusive behavior with increasing waiting time $t_{w}$. Further analysis of the distribution of particle displacements indicates that in the glassy state at short tracking times, the dynamics is heterogeneous. Using Mason's approximation [27] of the generalized Stokes-Einstein relation [28], we calculate the elastic and loss modulus from the MSD. With this microrheology technique, we probe the viscoelastic moduli in a lower frequency range, that is inaccessible with conventional macrorheology.

Quantitatively, however, we observe differences between the macro- and microrheology. To explain these differences we formulate a simple model for the frequency dependent drag force on the tracer particles, assuming that each tracer particle is surrounded by a liquid shell, which has viscoelastic properties that are different from the bulk.

\section{METHODS}

\section{A. Microgel particle suspension}

We use a suspension of thermosensitive core-shell particles with a polyNipam core and a polyNipmam shell [24-26]. The particles are swollen at low temperatures, $T$ $<30{ }^{\circ} \mathrm{C}$ and collapse at high temperatures, $T>45^{\circ} \mathrm{C}$, with a gentle transition around $35^{\circ} \mathrm{C}$. The radius of gyration, measured with static light scattering, varies by almost a factor of 2 in this temperature interval, as shown in Fig. 1. The suspension was prepared by adding a known amount of solvent (bidistilled water) to the freeze dried particles, resulting in a mass concentration of $4 \% w / w$ at $T_{0}=24{ }^{\circ} \mathrm{C}$. Carboxylated polystyrene spheres with a radius of $115 \mathrm{~nm}$, labeled with fluorescein (excitation wavelength: $490 \mathrm{~nm}$, emission: $540 \mathrm{~nm}$ ) were dispersed in the suspension to a concentration of $0.05 \% \mathrm{w} / \mathrm{w}$. The suspension was stirred over night to mix it homogeneously. The volume fraction of microgel particles in the suspension $\phi(T, c)$ was determined by measuring the relative viscosity $\eta_{r}$ at $T_{0}=24{ }^{\circ} \mathrm{C}$ as a function of the relative mass concentration $c$ in the low concentration regime: $c<5 \times 10^{-5} w / w$. The ratio $\phi / c$ was determined by applying Einstein's viscosity relation, $\eta_{r}(c)=1+5 / 2 \phi$, to the measured viscosities: $\phi\left(T_{0}, c\right) / c=42 \pm 1$. For other temperatures, the value of $\phi$ was calculated with $\phi(T, c)$ $=\phi\left(T_{0}, c\right)\left(R_{g}(T) / R_{g}\left(T_{0}\right)\right)^{3}$, where the particle gyration radius $R_{g}(T)$, was obtained from Fig. 1. All experiments were performed with volume fractions, as defined in this way, between 1.1 and 1.6.

\section{B. Particle tracking experiments}

To determine the displacement distributions and the MSDs of the fluorescent tracer particles as a function of time, we measure their displacements by taking series of images using a confocal scanning laser microscope (CSLM) equipped with a $100 \times$ objective and a CCD camera. The images are stored on disk for further analysis. To perform these experiments we need only two milliliter of sample, which is put together with a magnetic stirring bar, in a glass vial, from which the bottom has been removed, after which it was glued on to a Delta T culture dish (Bioptechs, Butler, PA, USA). Via this dish, the sample temperature is controlled using the accompanying delta $\mathrm{T}$ heater. To prevent sample evaporation, $1 \mathrm{ml}$ of mineral oil is added on top of the sample before tightly closing the vial.

The detection limit of the CSLM was determined by measuring the apparent displacement as a function of time of probe particles glued on the culture dish. They were glued by adding one drop of the probe suspension $(0.01 \% \mathrm{w} / \mathrm{w})$ and drying the dish in an oven at $80{ }^{\circ} \mathrm{C}$ for about four hours. The position of the particles was tracked using the CSLM by taking 2600 images at a rate of 1 frame per second (fps). From these measurements the smallest detectable in plane MSD was found: $5 \times 10^{-5} \mu \mathrm{m}^{2}$.

Before we start a measurement, the sample temperature is stabilized for about one hour. The surrounding temperature is kept at the same temperature as the sample using an infrared lamp. Next, to prepare a well defined initial state, the sample is stirred manually with the magnetic bar. The age of the sample is measured from the moment the stirring is stopped. Since we are interested in the age dependence of the particle dynamics, we wait a well defined time $t_{w}$, between 300 and $4500 \mathrm{~s}$, before we start to track the motion of the probe particles, by recording 2500 images of the sample with a rate of $1 \mathrm{fps}$ at $T=27^{\circ} \mathrm{C}$, and $10 \mathrm{fps}$ at higher temperatures.

The recorded images are analyzed using open source particle tracking routines written in interactive data language (IDL) from Research Systems Inc. to locate the position of the particles in every image [29]. Knowing the particle positions in each frame, we then construct the particle trajectories and calculate the ensemble-averaged two-dimensional mean squared displacements in the focal plane, using routines developed in the course of this project, that were written in " $C^{\prime \prime}$. Since the sample is also aging during recording, several 
waiting times can be considered by starting the analysis at a later recording time. For example, $t_{w}=800 \mathrm{~s}$ was obtained by calculating the MSD of the sample with $t_{w}=300 \mathrm{~s}$, but starting the analysis at 501st image, i.e., after an additional $500 \mathrm{~s}$. In order to minimize the contribution of the measurement time $t-t_{w}$ to the age of the sample, we considered only MSDs for $0<\left(t-t_{w}\right) / t_{w} \leq 0.4$ for the analysis of the MSD in the glassy state $\left(27^{\circ} \mathrm{C}\right)$.

The displacement distributions $P\left(y, t, t_{w}\right)$ during a certain time interval $\left[t_{w}, t\right]$ are determined by collecting the number of occurrences $y_{i}<\Delta r\left(t, t_{w}\right)<y_{i+1}$ into bin $\left[y_{i}, y_{i+1}\right]$. The number of occurrences in a bin is normalized by the total number of particles in the distribution times the bin width $y_{i+1}-y_{i}$.

\section{Microrheology}

The motion of the probe particle is determined by the (local) viscoelastic properties of the system. The relation between the complex shear modulus $G^{*}(\omega)$ and the twodimensional mean squared displacement $\left\langle\Delta r^{2}(t)\right\rangle$, is given by the Generalized Stokes-Einstein Relation (GSER) [28]

$$
G^{*}(\omega)=i \omega \widetilde{G}(i \omega)=\frac{2 k_{B} T}{3 \pi a(i \omega)\left\langle\widetilde{\Delta r^{2}}(i \omega)\right\rangle}
$$

Here, $\left\langle\widetilde{\Delta r^{2}}(s)\right\rangle$ is the Laplace transform of the $\operatorname{MSD}\left\langle\Delta r^{2}(t)\right\rangle$ and $\widetilde{G}(s)$ the Laplace transform of the viscoelastic relaxation modulus $G(t), k_{B}$ is the Boltzmann constant, $T$ is the thermodynamic temperature, $a$ is the radius of the probe particle and $i=(-1)^{1 / 2}$ the imaginary unit. Because in practice, $\left\langle\Delta r^{2}(t)\right\rangle$ is only known in a limited time regime, it is not possible to calculate its Laplace transform accurately. Therefore, we use Mason's approximation of the GSER [27]

$$
G^{*}(\omega)=\frac{2 k_{B} T}{3 \pi a\left\langle\Delta r^{2}(1 / \omega)\right\rangle} \frac{\exp \left[\frac{i \pi}{2} \alpha(\omega)\right]}{\Gamma[1+\alpha(\omega)]},
$$

where

$$
\alpha(\omega)=\left[\frac{d \ln \left\langle\Delta r^{2}\left(t-t_{w}\right)\right\rangle}{d \ln \left(t-t_{w}\right)}\right]_{t-t_{w}=\omega^{-1}}
$$

is the slope of the log-log plot of $\left\langle\Delta r^{2}\right\rangle$ vs $\left(t-t_{w}\right)$ and $\Gamma$ is the so-called gamma function, which for $1 \leq z \leq 2$, corresponding to $0 \leq \alpha \leq 1$, is well approximated by: $\Gamma(z) \approx 0.457 z^{2}$ $-1.36 z+1.90$. The elastic and the loss modulus, which are the real and the imaginary part of $G^{*}(\omega)$, are given by

$$
\begin{aligned}
G^{\prime}(\omega) & =\frac{2 k_{B} T}{3 \pi a\left\langle\Delta r^{2}\left(\omega^{-1}\right)\right\rangle} \frac{\cos [\pi \alpha(\omega) / 2]}{\Gamma[1+\alpha(\omega)]}, \\
G^{\prime \prime}(\omega) & =\frac{2 k_{B} T}{3 \pi a\left\langle\Delta r^{2}\left(\omega^{-1}\right)\right\rangle} \frac{\sin [\pi \alpha(\omega) / 2]}{\Gamma[1+\alpha(\omega)]} .
\end{aligned}
$$

To reduce the uncertainty in the derivatives needed to calculate $\alpha(\omega)$, we approximated the measured MSD curves by an empirical function $f(x)=\left[\left(a x^{n}\right)^{p}+\left(b x^{m}\right)^{p}\right]^{1 / p}$, where $x=t-t_{w}$. The terms $a x^{n}$ with $n \simeq 0^{+}$and $b x^{m}$ with $m \simeq 1^{-}$describe the

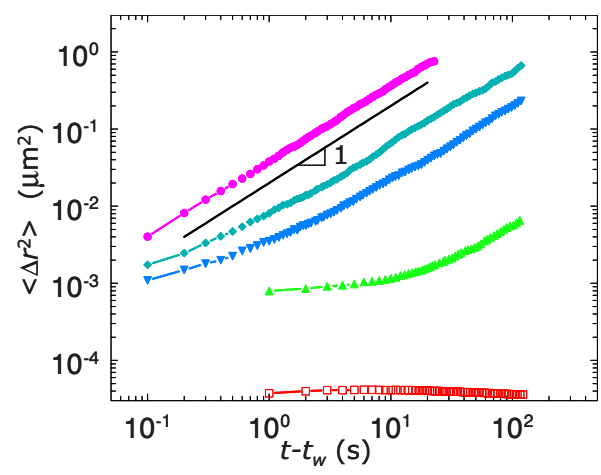

FIG. 2. (Color online) The mean squared displacement $\left\langle\Delta r^{2}\right\rangle$ of the probe particles measured for $c=4 \% \mathrm{w} / \mathrm{w}$ at different temperatures (from top to bottom $32{ }^{\circ} \mathrm{C}, 31{ }^{\circ} \mathrm{C}, 30^{\circ} \mathrm{C}$, and $27{ }^{\circ} \mathrm{C}$, with $\phi=1.18,1.28,1.37$, and 1.56 , respectively) and $t_{w}=300 \mathrm{~s}$. The open squares indicate the detection limit of our CSLM setup.

short and long time behavior of the curve, while $p$ is a measure for the smoothness of the transition from short to long time behavior, which occurs at $a x^{n}=b x^{m}$. The coefficients $a$, $b, n, m$, and $p$ are obtained by fitting $f(x)$ to the experimental data. The derivatives are calculated by differentiating $f(x)$. Finally, the viscoelastic moduli are calculated using Eqs. (3)-(5).

\section{Macrorheology}

For comparison with the microrheology results, we also measured the macroscopic elastic and loss modulus, $G^{\prime}$ and $G^{\prime \prime}$, of the suspension in a frequency range of $0.063-6.28$ $\mathrm{rad} / \mathrm{s}$, using a Haake RS600 rheometer with a cone and plate geometry (cone angle: 2 degrees, diameter: $60 \mathrm{~mm}$ ). A home built vapor lock was used to avoid evaporation. The temperature of the shielding was kept approximately $5{ }^{\circ} \mathrm{C}$ above the plate temperature to prevent condensation on it. This was sufficient to keep the concentration constant for more than a week. The suspension was injected at about $44{ }^{\circ} \mathrm{C}$ (collapsed state) and then the instrument was cooled down to the experimental temperature. Prior to any oscillatory measurement, the suspension was rejuvenated by a mechanical quench, i.e., a stress well above the yield stress was applied for $60 \mathrm{~s}$. The time $t=0$ is defined at the end of the quench. The elastic modulus $G^{\prime}(\omega)$ and the loss modulus $G^{\prime \prime}(\omega)$ were measured at several temperatures $T$ and after different waiting times $t_{w}$. The age is defined as the total time since the end of the mechanical quench until the moment of data acquisition, which includes the waiting time and the oscillation time so far $[4,5]$.

\section{PARTICLE TRACKING RESULTS}

\section{A. Mean square displacements}

The two-dimensional mean squared displacements of the probe particles embedded in a $4 \% \mathrm{w} / \mathrm{w}$ suspension are measured, at different temperatures, as a function of the tracking time $t-t_{w}$ after a waiting time $t_{w}=300 \mathrm{~s}$. The results are shown in Fig. 2 using logarithmic scales. The MSD curves behave almost linearly for $T>30{ }^{\circ} \mathrm{C}$, indicating the liquid 


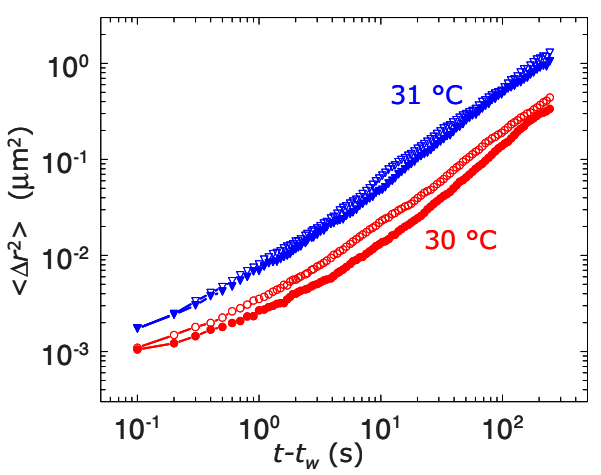

FIG. 3. (Color online) The MSD $\left\langle\Delta r^{2}\right\rangle$ as a function of time at $30{ }^{\circ} \mathrm{C}(\bigcirc, \phi=1.37)$ and $31{ }^{\circ} \mathrm{C}(\nabla, \phi=1.28)$ measured after $t_{w}$ $=300 \mathrm{~s}$ (open symbols) and $t_{w}=3000 \mathrm{~s}$ (filled symbols).

like behavior of the suspension at these temperatures. For $T \leq 30{ }^{\circ} \mathrm{C}$, the curves show a transition from liquid like to glassy, reflected by the onset of a plateau at short times. Moreover, the curves shift downwards as the temperature decreases. Note that the MSDs measured at different temperatures are well above the detection limit, $5 \times 10^{-5} \mu \mathrm{m}^{2}$ that was determined from the apparent MSD of probe particles glued to the culture dish (the lowest curve in Fig. 2).

At $32{ }^{\circ} \mathrm{C}$, the MSD increases linearly with tracking time as indicated by the slope of the $\left\langle\Delta r^{2}\left(t-t_{w}\right)\right\rangle$ curve, which is close to one, whereas at lower temperature $\left(30^{\circ} \mathrm{C}\right.$ and $31{ }^{\circ} \mathrm{C}$ ) the slope for $t-t_{w}<2 \mathrm{~s}$ is smaller than unity, tending to unity at longer tracking times. At $27{ }^{\circ} \mathrm{C}$, the MSD curve shows a plateau at short tracking times $\left(t-t_{w}<10 \mathrm{~s}\right)$ while it increases linearly with the tracking time for $t-t_{w}>20 \mathrm{~s}$. At long tracking times, the behavior of the MSD curves is diffusive in all cases and the suspension behaves liquidlike. In this regime, the diffusivity goes down with decreasing temperature. This decrease is determined by the low frequency viscosity $\eta_{0}^{\prime}$ of the suspension, $\left\langle\Delta r^{2}\right\rangle /\left(t-t_{w}\right) \simeq 2 k_{B} T / 3 \pi a \eta_{0}^{\prime}$, which increases with decreasing temperature due to the increasing volume fraction. A similar behavior of the mean square displacement has been found in molecular dynamic simulations of dense suspensions of star polymers. However, for star polymers the size of the particles increases as the temperature increases [30].

At temperatures below $30{ }^{\circ} \mathrm{C}$ and short tracking times, subdiffusive behavior is observed, i.e., the slope of the $\ln \left\langle\Delta r^{2}\right\rangle$ vs $\ln \left(t-t_{w}\right)$ curve is lower than one. This reflects the onset of elastic behavior [27], which is most pronounced at $27^{\circ} \mathrm{C}$. Here, the MSD shows a plateau at short time scales because the particles are trapped within a cage formed by their neighboring particles, and the system behaves elastically. The relation between the elastic modulus, $G_{\infty}^{\prime}$, and the plateau value of the MSD, $\left\langle\Delta r^{2}\right\rangle_{p}$ is given by $G_{\infty}^{\prime}$ $\simeq\left(2 k_{B} T / 3 \pi a\right) \times\left(1 /\left\langle\Delta r^{2}\right\rangle_{p}\right)$.

To identify the transition from the (nonaging) liquid to the (aging) glassy state, we measure the MSD curves at $31{ }^{\circ} \mathrm{C}$ and $30^{\circ} \mathrm{C}$, at two different waiting times $\left(t_{w}=300\right.$ and 3000 s). The results are given in Fig. 3. The MSDs measured at both temperatures look quite similar. But at $31{ }^{\circ} \mathrm{C}$, we observe no significant difference (considering the reproducibility of the MSD measurements) between the curves measured
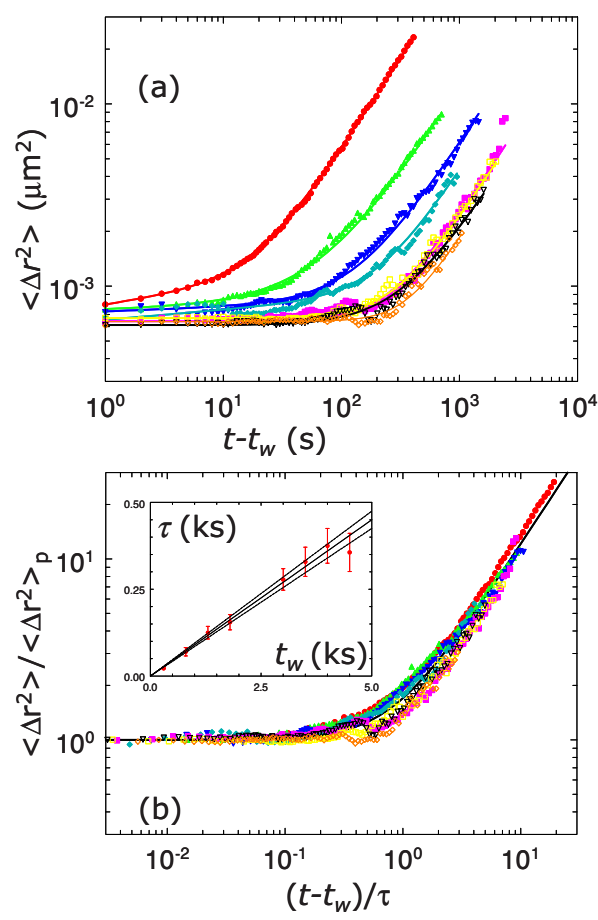

FIG. 4. (Color online) Panel a: the MSD $(c=4 \% w / w$ and $T$ $\left.=27{ }^{\circ} \mathrm{C}, \phi=1.56\right)$ measured at several waiting times [300 s (filled $\bigcirc), 800 \mathrm{~s}$ (filled $\triangle$ ), $1300 \mathrm{~s}$ (filled $\nabla$ ), $1800 \mathrm{~s}$ (filled $\diamond), 3000 \mathrm{~s}$ (filled $\square$ ), $3500 \mathrm{~s}$ (open $\square$ ), $4000 \mathrm{~s}$ (open $\nabla$ ), and $4500 \mathrm{~s}$ (open $\diamond)$ ]. Panel b: the same data but the MSDs and $t-t_{w}$ have been normalized with $\left\langle\Delta r^{2}\right\rangle_{p}$ and the crossover time $\tau$, respectively. Inset: $\tau$ as a function of the waiting time $t_{w}$ in units of $1000 \mathrm{~s}$.

at $t_{w}=300 \mathrm{~s}$ and $t_{w}=3000 \mathrm{~s}$, while at $30{ }^{\circ} \mathrm{C}$, the MSD does depend on the waiting time. Hence, the transition temperature $T_{g}$ of the $4 \% w / w$ suspension lies between $30{ }^{\circ} \mathrm{C}$ and $31{ }^{\circ} \mathrm{C}$ This is in agreement with our previous observations where we found for $c=4 \% w / w: T_{g}=29^{\circ} \mathrm{C}$, see Fig. 3 of [6].

The dependence of the MSD curves on the waiting time becomes more pronounced at lower temperatures, where the volume fraction of the system is larger. Therefore, we reduced the temperature to $27{ }^{\circ} \mathrm{C}$ and measured the ensemble averaged MSD as a function of the tracking time for eight different waiting times between 300 and $4500 \mathrm{~s}$. The results are given in Fig. 4(a). The figure shows clearly the evolution of the MSD with increasing waiting time $t_{w}$ : the transition from the short time plateau to the long time diffusive growth of the MSD, characterized by the crossover time $\tau$, shifts towards longer times, while the plateau values themselves slightly decrease with increasing $t_{w}$. This result strongly indicates the aging of the system. In Fig. 4(b), we scale the results by normalizing the MSD with its plateau value $\left\langle\Delta r^{2}\right\rangle_{p}$, and plotting them as function of $\left(t-t_{w}\right) / \tau$. The crossover time $\tau$ is determined from the transition from the plateau to the diffusive part of the MSD, using the coefficients of the empirical fit function mentioned in Sec. II C: $a \tau^{n}$ $=b \tau^{m}$. The inset of Fig. 4(b) shows the crossover time $\tau$ as a function of the waiting time $t_{w}$. A linear fit to these data points results in: $\tau / t_{w}=0.090 \pm 0.005$ : the crossover time increases linearly with the age of the suspension. 


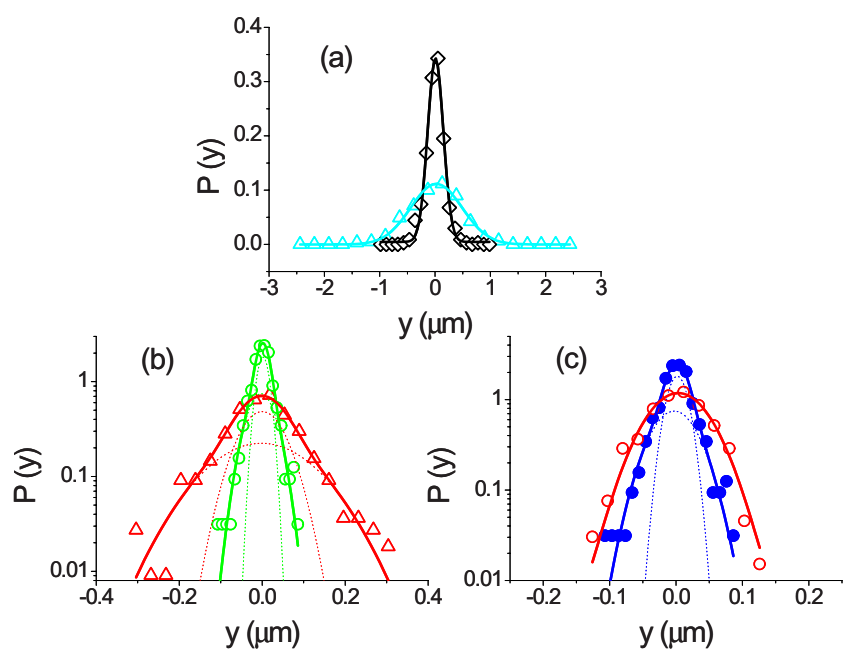

FIG. 5. (Color online) Panel a: the displacement probability $[P(\Delta y)]$ at $31{ }^{\circ} \mathrm{C}(\diamond, \phi=1.28)$ and $32{ }^{\circ} \mathrm{C}(\triangle, \phi=1.18)$ taken at $t_{w}=300 \mathrm{~s}$ and $t-t_{w}=10 \mathrm{~s}$ and compared to Gaussian fits (lines). Panel b: $P(\Delta y)$ at $27{ }^{\circ} \mathrm{C}(\bigcirc, \phi=1.56)$ and $30{ }^{\circ} \mathrm{C}(\triangle, \phi=1.37)$ taken at $t_{w}=300 \mathrm{~s}$ and $t-t_{w}=10 \mathrm{~s}$ and compared to a double Gaussian (lines). The dotted lines indicate the mobile and immobile populations. Panel c: $[P(\Delta y)]$ at $27{ }^{\circ} \mathrm{C}(\phi=1.56)$ taken at $t_{w}$ $=300 \mathrm{~s}$ and $t-t_{w}=10 \mathrm{~s}$ (closed symbols) and $t-t_{w}=100 \mathrm{~s}$ (open symbols). At $t-t_{w}=10 \mathrm{~s}, P(\Delta y)$ is well described with a double Gaussian while at $t-t_{w}=100 \mathrm{~s}$, can be described with one Gaussian.

\section{B. Displacement distributions}

Analyzing the particle trajectories we also constructed the displacement distributions $P\left(\Delta x ; t, t_{w}\right)$ and $P\left(\Delta y ; t, t_{w}\right)$, where $\Delta x=x(t)-x\left(t_{w}\right)\left[\Delta y=y(t)-y\left(t_{w}\right)\right]$ is the displacement in the focal plane in $x(y)$ direction. Figure 5(a) shows the displacement distribution of the probe particles $P\left(\Delta y ; t, t_{w}\right)$ at $31{ }^{\circ} \mathrm{C}$ and $32{ }^{\circ} \mathrm{C}$, when the system is in the liquid state, taken at $t_{w}=300 \mathrm{~s}$ and $\Delta t=t-t_{w}=10 \mathrm{~s}$. At these temperatures, the distributions are Gaussian, i.e., $P(\Delta y) \sim \exp ($ $\left.-\Delta y^{2} / 2\left\langle\Delta y^{2}\right\rangle\right)$, as expected for a liquid. The displacement distribution at $32{ }^{\circ} \mathrm{C}$ is broader than the one at $31{ }^{\circ} \mathrm{C}$, because the probe particles have a higher diffusivity at $32{ }^{\circ} \mathrm{C}$, as shown in Fig. 2. This is due to a lower viscosity. The viscosities at $32{ }^{\circ} \mathrm{C}$ and $31{ }^{\circ} \mathrm{C}$, calculated using StokesEinstein relation: $\eta \simeq\left(2 k_{B} T / 3 \pi a\right) \times\left(\left(t-t_{w}\right) /\left\langle\Delta r^{2}\right\rangle\right)$, are 0.2 Pas and 1.2 Pas, respectively.

The displacement distributions presented in Fig. 5(a) include all the probe particles in the observation window. Every probe particle explores randomly its local environment resulting in a Gaussian displacement distribution. Averaging over all the particles will reveal the homogeneity of the sample, since any spatial inhomogeneity will lead to a nonGaussian displacement distribution. On the other hand, a Gaussian distribution shows that the sample is homogeneous, at least on the length scale of the observation window.

Figure 5(b) shows the displacement distribution of the probe particles when the suspension is in the glassy state $\left(t_{w}=300 \mathrm{~s}\right)$, at $30{ }^{\circ} \mathrm{C}$ just below the glass transition and at $27{ }^{\circ} \mathrm{C}$ deeper in the glassy state. The distribution at $30{ }^{\circ} \mathrm{C}$ is broader than the one at $27^{\circ} \mathrm{C}$ where the system is more

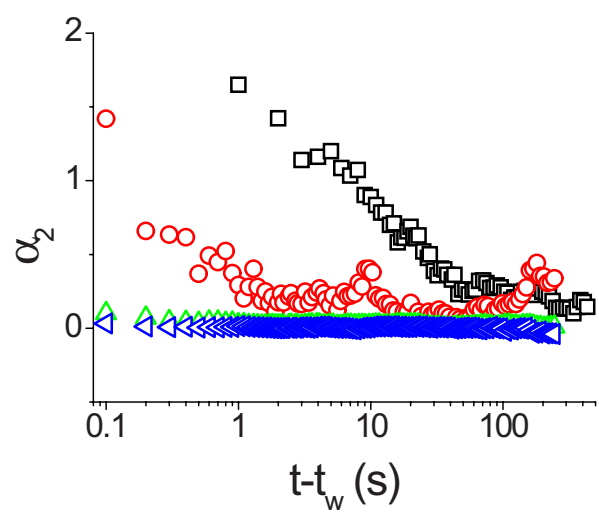

FIG. 6. (Color online). The non-Gaussian parameter $\alpha_{2}\left(t_{w}\right.$ $=300 \mathrm{~s})$ at several temperatures: $27{ }^{\circ} \mathrm{C}(\square, \phi=1.56), 30{ }^{\circ} \mathrm{C}(\bigcirc$, $\phi=1.37), 31{ }^{\circ} \mathrm{C}(\triangle, \phi=1.28)$, and $32{ }^{\circ} \mathrm{C}(\triangleleft, \phi=1.18)$.

arrested and the probe particles have less freedom to move. In contrast to the distributions in the liquid state, these distributions are non-Gaussian and can be described by a double Gaussian as shown by the solid line in Fig. 5(b). The two underlying Gaussian distributions are indicated by the dashed lines. The non-Gaussian behavior observed in the glassy state indicates a dynamic inhomogeneity as we perform ensemble averaging only $[1,7]$, so all particles have the same age. The double Gaussian observed in Fig. 5(b), suggests that in the glassy state, two populations of particles exist with different dynamic behavior. The broader Gaussian distribution represents the mobile population, whereas the less mobile population is represented by the more narrow Gaussian.

In Fig. 5(c), we consider the suspension at $27{ }^{\circ} \mathrm{C}$ and $t_{w}$ $=300 \mathrm{~s}$. The displacement distributions are taken at two different tracking times: $t-t_{w}=10$ and $100 \mathrm{~s}$. At $t-t_{w}=10 \mathrm{~s}$, the particles are caged by the neighboring particles as indicated by the almost constant $\left\langle\Delta r^{2}\right\rangle$ shown in Fig. 4(a), whereas at $t-t_{w}=100 \mathrm{~s}$, the particles behave diffusive. The distribution is broader at longer $t-t_{w}$ as also indicated by the increase in its MSD shown in Fig. 2. The displacement in the caged part $\left(t-t_{w}=10 \mathrm{~s}\right)$ can be well described with a double Gaussian, whereas a single Gaussian is sufficient to describe the distribution at $t-t_{w}=100 \mathrm{~s}$.

To investigate how this distribution of the displacement evolves as function of $t-t_{w}$, we quantify the deviation from a Gaussian distribution by calculating the non-Gaussian parameter [1]: $\alpha_{2}=\frac{1}{3}\left\langle\Delta r^{4}\left(t-t_{w}\right)\right\rangle /\left\langle\Delta r^{2}\left(t-t_{w}\right)\right\rangle^{2}-1$. Figure 6 shows $\alpha_{2}$ for the $4 \% w / w$ microgel suspension at different temperatures. The analysis is done at $t_{w}=300 \mathrm{~s}$. At high temperature $\left(31^{\circ} \mathrm{C}\right.$ and $\left.32{ }^{\circ} \mathrm{C}\right)$, the $\alpha_{2}$ is zero for all observed time scales. However, at low temperatures $\left(27^{\circ} \mathrm{C}\right.$ and $30{ }^{\circ} \mathrm{C}$ ), the non-Gaussian parameter is larger than zero at short time scales and decreases to zero at longer time scales. We also observe that the non-Gaussian parameter reduces to zero within a relatively short time $\left(t-t_{w} \sim 1 \mathrm{~s}\right)$ at $30^{\circ} \mathrm{C}$ while at $27{ }^{\circ} \mathrm{C}$, it takes about $100 \mathrm{~s}$. Figure 6 indicates that $\alpha_{2}$ is nonzero when the particles are caged and reduces to zero once the particles escape from the cage. This evolution of the displacement probability from non-Gaussian at short times to Gaussian at long times is not in agreement with a 


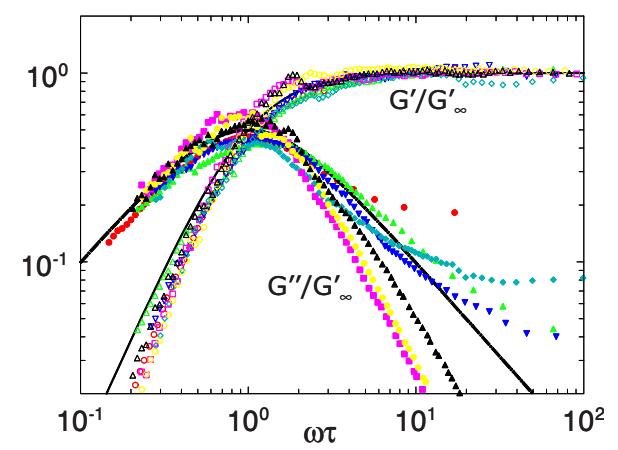

FIG. 7. (Color online) The normalized $G^{\prime}$ (open symbols) and the $G^{\prime \prime}$ (filled symbols) of a $4 \% w / w$ suspension $\left(T=27^{\circ} \mathrm{C}, \phi\right.$ $=1.56)$ at several waiting times $[300 \mathrm{~s}(\bigcirc), 800 \mathrm{~s}(\triangle), 1300 \mathrm{~s}(\nabla)$, $1800 \mathrm{~s}(\diamond), 3000 \mathrm{~s}(\square), 3500 \mathrm{~s}(\bigcirc), 4000 \mathrm{~s}(\triangle)$, and $4500 \mathrm{~s}(\nabla)]$ calculated from Fig. 4, using Mason's approximation of the Generalized Stokes-Einstein Relation.

recent theory developed based on a single Brownian particle moving in a periodic effective field [31], which describes the caging and subsequent cage escape. This theory correctly predicts the Gaussian displacement distribution in the diffusive region of the MSD curve. However, it predicts also Gaussian behavior at the plateau region of the MSD, only near the transition the model predicts a non-Gaussian distribution, resulting in a maximum in the $\alpha_{2}$ vs $t-t_{w}$ curve. Because we do observe non-Gaussian behavior at the plateau region, which cannot be attributed to the caging effect, as [31] suggests, we conclude this is most probably due to spatial inhomogeneity of the sample.

\section{Microrheology}

To convert the data from Fig. 4 to the viscoelastic moduli $G^{\prime}$ and $G^{\prime \prime}$, we determine $\alpha(\omega)$, Eq. (3), for every MSD curve as described in Sec. II C and calculate the moduli using Eq. (4) and (5). The results are shown in Fig. 7, where $G^{\prime}$ and $G^{\prime \prime}$, both scaled on $G_{\infty}^{\prime}$, have been plotted vs $\omega \tau$, with $\tau$ the crossover time from Fig. 4. All $G^{\prime}$ and $G^{\prime \prime}$ curves collapse to a master curve. The full lines in the figure represent a Maxwell fluid which has only one relaxation time. As the crossing of $G^{\prime}$ and $G^{\prime \prime}$ occurs at $\omega \tau=1$, we identify the crossover as the mean relaxation time of the suspension. Hence, the mean relaxation time depends linearly on the age of the system: $\tau=0.1 t_{w}$. The relaxation time obtained for $t_{w}$ $=4500 \mathrm{~s}$, shown in the inset of Fig. 4(b), seems to be smaller than expected from the linear dependence. However, earlier macrorheology measurements on the same system at $T$ $=20{ }^{\circ} \mathrm{C}$ show that the relaxation times scale with age at least up to $t=30000 \mathrm{~s}$, see Fig. 4(a) of [6]. From the behavior of $G^{\prime \prime}$ at $\omega \tau \simeq 10$ we observe that $G^{\prime \prime}$ deviates from Maxwellian behavior for short waiting times. The deviation becomes smaller with increasing waiting time. This indicates that at short waiting times additional relaxation times exist, shorter than the dominant relaxation time, which disappear with increasing waiting time. Moreover, the low frequency behavior of $G^{\prime}$ (as well as the high frequency behavior of $G^{\prime \prime}$ at long waiting times) is steeper than prescribed by linear viscoelastic theory. This is most probably due to the limitations of the
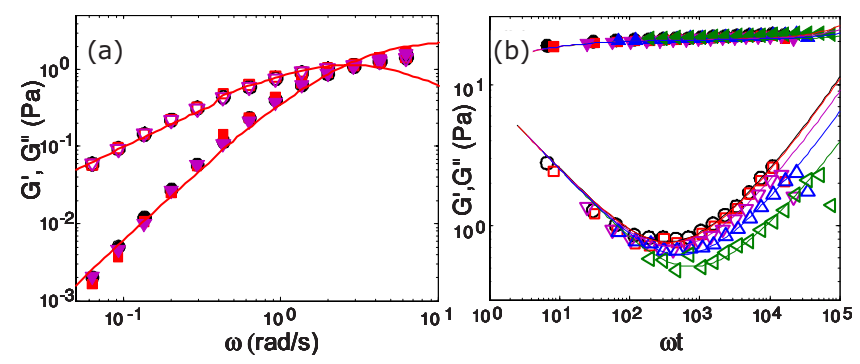

FIG. 8. (Color online) Elastic (filled symbols) and loss modulus (open symbols) measured at $32{ }^{\circ} \mathrm{C}$ (a) and $27{ }^{\circ} \mathrm{C}$ (b) corresponding to $\phi=1.18$ and 1.56 , respectively. The waiting times are: $3 \mathrm{~s}(\bigcirc), 30$ s $(\square), 300 \mathrm{~s}(\nabla), 1000 \mathrm{~s}(\triangle)$, and $3000 \mathrm{~s}(\triangleleft)$. The lines represent the SGR predictions. At $32{ }^{\circ} \mathrm{C}$, the moduli have been plotted vs $\omega$ and at $27{ }^{\circ} \mathrm{C}$ vs $\omega t$, to obtain a collapse to master curves. (Panel b, for $\omega t>200$, see text.)

Mason approximation [27], used to calculate the viscoelastic moduli.

\section{MICRO- VS MACRORHEOLOGY}

\section{A. Macroscopic viscoelastic moduli}

For comparison with the microrheology results, we also measured the macroscopic viscoelastic moduli, $G^{\prime}$ and $G^{\prime \prime}$, of the suspension in a frequency range of $0.062-6.28 \mathrm{rad} / \mathrm{s}$, as described before in [5]. Figure 8 shows the elastic and the loss modulus of the $4 \% \mathrm{w} / \mathrm{w}$ suspension measured at $32{ }^{\circ} \mathrm{C}$ and $27^{\circ} \mathrm{C}$, after several waiting times. From Fig. 8(a), we observe that at $32{ }^{\circ} \mathrm{C}$, corresponding with a volume fraction $\phi=1.18$, the loss modulus is larger than the elastic modulus for most frequencies and only at frequencies higher than 3 $\mathrm{rad} / \mathrm{s}$ the elastic modulus is larger than the loss modulus. The moduli are age-independent at this temperature as indicated by the collapse of the moduli measured at different waiting times. Figure $8(\mathrm{~b})$ shows that at $27{ }^{\circ} \mathrm{C}$, corresponding with $\phi=1.56$, the elastic modulus $G^{\prime}$ is almost constant and significantly larger than the loss modulus $G^{\prime \prime}$ for all applied frequencies. Moreover, at $27{ }^{\circ} \mathrm{C}$ the moduli depend on the age of the suspension. $G^{\prime}$ and $G^{\prime \prime}$ form a master curve for $\omega t<200$ when they are plotted as function of $\omega t$. Here, $t$ is the age of the sample at the moment of measuring the considered data point. For $\omega t>200$, the loss moduli $G^{\prime \prime}(\omega t)$ do not collapse to a single curve anymore. This is due to the dominance of local viscous and Brownian contributions, which are age-independent [4,5].

The transition from nonaging viscous behavior at high temperature [Fig. 8(a)] to aging elastic behavior at low temperature [Fig. 8(b)] again shows that the system undergoes a transition from liquid to glassy as we increase the volume fraction by decreasing the temperature. However, in this case, we cannot determine the mean relaxation time $\tau$, due to the limited experimental $\omega t$ window. By quantitatively comparing the viscoelastic moduli with the predictions of the soft glassy rheology (SGR) model as indicated by the lines in Fig. 8 (see [14-16] for details), we found that the system is in the liquid state at $32{ }^{\circ} \mathrm{C}$ with an effective noise temperature well above one: $x>3$. On the other hand, at $27^{\circ} \mathrm{C}$, we 


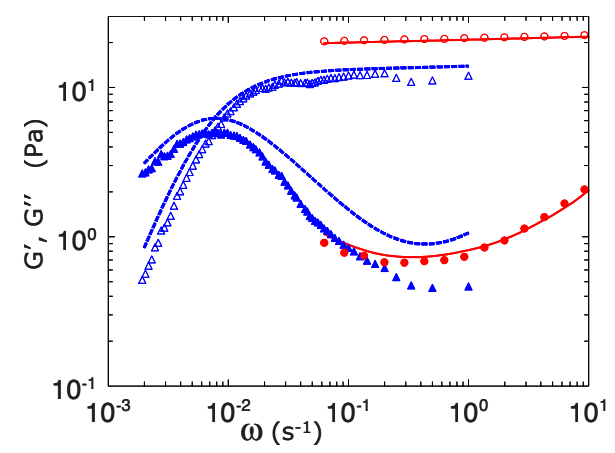

FIG. 9. (Color online) Comparison between the macro- $(\bigcirc)$ and microrheology $(\triangle)$ at $T=27{ }^{\circ} \mathrm{C}(\phi=1.56)$ and $t_{w}=1300 \mathrm{~s}$. The open (filled) symbols represent the measured $G^{\prime}\left(G^{\prime \prime}\right)$ values. The blue dashed curves are the results of the calculation, described in Sec. IV B, using the analytic representation of the macro results (full red lines) as input.

obtain an effective noise temperature well below one: $x$ $=0.5$, which means that the system is in the glassy state and ages. Also, according to the SGR model $G^{*}$ in the glassy state is a function of $\omega t$. This implies that all the underlaying relaxation times scale with the age of the system. So, the observed scaling of the mean relaxation time in Fig. 4(b) is qualitatively in agreement with the predictions of the SGR model.

To compare the macro- and microrheology directly, we plot in Fig. 9 the macroscopic (red circles) and local microscopic (blue triangles) $G^{\prime}$ and $G^{\prime \prime}$, both measured at $27{ }^{\circ} \mathrm{C}$ and after waiting a time $t_{w}=1300 \mathrm{~s}$. With Mason's approach, one "measures" the moduli in the frequency range between $\omega_{\min }=1 / \Delta t_{\max }$ and $\omega_{\max }=1 / \Delta t_{\min }$ where $\Delta t=t-t_{w}$ is the tracking time. In this range, the crossing of $G^{\prime}$ and $G^{\prime \prime}$, inaccessible in macrorheological measurements, is observed. Although both sets of curves show globally the same behavior, there are significant differences. The $G^{\prime}$ obtained from macrorheology is a factor 1.8 larger than the $G^{\prime}$ from microrheology. Moreover, the slopes of both $G^{\prime \prime}$ curves in the overlapping frequency range do not match indicating a shift in the relaxation time: $\tau_{\text {macro }} / \tau_{\text {micro }} \approx 5$. These differences occur because the probe particles feel their local environment. The properties of this environment will differ from the macroscopic bulk properties [32]. In the next section, we try to quantify this effect.

Another explanation found in literature [33] for the lower moduli measured using particle tracking is the possible existence of an effective temperature, which should be about two times the thermodynamic temperature. In this picture, the macrorheology measurement is considered as the response of the system to an external force (equivalent with probing mobility) and the microrheology is obtained from the displacements of the probe particles embedded in the system (probing diffusivity). But this cannot explain the rather large shift in the mean relaxation time.

\section{B. Local drag force on a tracer particle}

The size of the tracer particle is of the order of the microgel particles. In that case, one may expect that the tracer

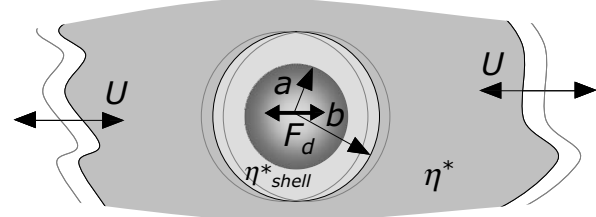

FIG. 10. Cell model to calculate the drag force $F_{d}$ on the probe particle with radius $a$, due to an oscillating velocity field $U$. The surrounding shell, with viscosity $\eta_{\text {shell }}^{*}$, has a radius $b(\beta=b / a)$.

probes an environment, where the viscoelasticity is determined by the local polymer concentration near the surface of the microgel particle. This concentration can be considerably lower than at the core of such a microgel particle $[34,35]$. Hence, the local elasticity and viscosity are expected to be much smaller than the bulk values. To investigate this effect, we model the local environment as a spherical shell with radius $b$ around the probe particle with radius $a$ (see Fig. 10). This shell has a complex viscosity $\eta_{\text {shell }}^{*}$, which is different from the bulk. Outside the shell, we assume the macroscopic viscoelasticity $G^{*}(\omega)=i \omega \eta^{*}(\omega)$. For this situation, the drag force $F_{d}$ on a stationary tracer particle in the frequency domain is not simply given by $F_{d}(\omega)=6 \pi a \eta^{*}(\omega) U(\omega)$ where $U$ is the fluid velocity far from the tracer, but will also be influenced by the viscoelastic shell around the tracer. We calculate the modified drag force by considering a stationary particle, surrounded by a viscoelastic shell (region 1), in a viscoelastic medium (region 2) that moves with velocity $U(\omega)$. As described in the supplementary information [36], we ignore inertia and solve the Stokes equations with the appropriate boundary conditions [37]

$$
\nabla \cdot \underline{u}=0, \quad \nabla p=\mu^{*} \nabla^{2} \underline{u},
$$

where $\underline{u}$ is the velocity field, $p$ the pressure field, and $\mu^{*}$ the complex viscosity in the considered region. The boundary conditions far away from the particle in region 2 are given by the applied velocity field

$$
u_{r}^{[2]}(r, \vartheta)=U \cos \vartheta, \quad u_{\vartheta}^{[2]}(r, \vartheta)=-U \sin \vartheta
$$

while at the particle surface in region 1 , i.e., $r=a$, the velocity components should be zero

$$
u_{r}^{[1]}(r, \vartheta)=u_{\vartheta}^{[1]}(r, \vartheta)=0
$$

and at the boundary between region 1 and 2 , i.e., $r=b$, the velocities and tractions should be continuous

$$
\begin{array}{ll}
u_{r}^{[1]}(r, \vartheta)=u_{r}^{[2]}(r, \vartheta), & T_{r r}^{[1]}=T_{r r}^{[2]}, \\
u_{\vartheta}^{[1]}(r, \vartheta)=u_{\vartheta}^{[2]}(r, \vartheta), & T_{r \vartheta}^{[1]}=T_{r \vartheta}^{[2]},
\end{array}
$$

Having solved the velocity and pressure field, we integrate the resulting stresses $T_{r r}^{[1]}$ and $T_{r \vartheta}^{[1]}$ over the surface of the tracer particle to find the drag force on the tracer

$$
F_{d}(\omega)=6 \pi a Q\left(\beta, \eta^{*} / \eta_{\text {shell }}^{*}\right) \eta^{*}(\omega) U(\omega),
$$

where $\beta=b / a$ is the relative outer radius of the shell surrounding the tracer. The function $Q\left(\beta, \eta_{r}\right)$ is defined as 


$$
Q\left(\beta, \eta_{r}\right)=\frac{A_{0}(\beta)+\eta_{r} A_{1}(\beta)}{B_{0}(\beta)+\eta_{r} B_{1}(\beta)+\eta_{r}^{2} B_{2}(\beta)},
$$

with

$$
\begin{gathered}
A_{0}(\beta)=6 \beta^{6}+4 \beta, \\
A_{1}(\beta)=4 \beta^{6}-4 \beta, \\
B_{0}(\beta)=6 \beta^{5}+4, \\
B_{1}(\beta)=6 \beta^{6}+3 \beta^{5}-10 \beta^{3}+9 \beta-8, \\
B_{2}(\beta)=4 \beta^{6}-9 \beta^{5}+10 \beta^{3}-9 \beta+4 .
\end{gathered}
$$

Note that both $Q\left(1, \eta_{r}\right)=1$ and $Q(\beta, 1)=1$, as expected. Once this force is known, we can write the local viscoelasticity felt by the tracer particle as $6 \pi a \eta_{\mathrm{pt}}^{*}(\omega) U(\omega)$ $=6 \pi a Q\left(\beta, \eta^{*} / \eta_{\text {shell }}^{*}\right) \eta^{*}(\omega) U(\omega)$ or

$$
G_{\mathrm{pt}}^{*}(\omega)=Q\left(\beta, \eta^{*} / \eta_{\text {shell }}^{*}\right) G^{*}(\omega) .
$$

In Fig. 9, we compare the measured $G_{\mathrm{pt}}^{*}(\omega)$ with those calculated from $G^{*}(\omega)$ using last equation (blue solid line). Since the segment density profile of the microgel particles, as investigated previously [24], show a smooth decay at the particle surface, the crosslink density at the microgel surface is very low. Hence, the microgel surface is dominated by the dangling chains and thus should reveal mainly viscous properties. We therefore assume $\eta_{\text {shell }}^{*}=\eta_{\infty}^{\prime}$. The parameter $\beta$ was used as a fitting coefficient. The best match was found for $\beta=1.05$. For this value, we obtain $G^{*}(\infty) / G_{\mathrm{pt}}^{*}(\infty) \simeq 1.6$ and $\tau_{\text {macro }} / \tau_{\text {micro }} \simeq 5$, which is qualitatively in agreement with the experimental observation. Although the model is very simple and certainly not adequate to describe all details, it clearly shows that a small depletion region around the tracer particle already causes a drastic change in its mobility.

\section{CONCLUSION}

With our thermosensitive system, we can tune the system from the liquid to the glassy state reversibly by changing the temperature. The volume fraction of the system increases as we decrease the temperature due to the swelling of the coreshell particles. The viscoelastic moduli obtained from macrorheology evolve from a viscous behavior to an elastic behavior as we decrease the temperature. Meanwhile, the mean squared displacement obtained from the particle tracking experiments evolves from a diffusive at high temperature to a caging-diffusive behavior at low temperature. In the glassy state (at low temperature), the system shows aging as indicated by the age-dependent behavior of the viscoelastic moduli obtained from macrorheology and the MSD curves obtained from the particle tracking. With this particle tracking technique, we are able to measure directly the mean relaxation time of an aging suspension. The relaxation time extracted from these measurements increases linearly with the waiting time, i.e., the age of the sample, and is qualitatively in agreement with the indirect macrorheological observation that the moduli measured at different ages form a master curve when plotted as function of $\omega t$ in stead of $\omega$, implying a linear increase in the relaxation times with age $t$.

Investigation of the displacement distributions indicates that in the glassy state, the particle dynamics is non-Gaussian at short tracking times but becomes Gaussian at longer times. The non-Gaussian behavior indicates that the particle displacements are inhomogeneous. At short tracking times, we identify in the glassy state mobile and immobile particle populations.

Using Mason's approximation of the generalized StokeEinstein relation, we calculate the viscoelastic moduli from the measured MSDs. Comparing the macro- and microrheology quantitatively, we observe a shift both in the elastic plateau and the mean relaxation time, which we can explain by a simple model calculation that takes into account the local inhomogeneity of the sample around the tracer particle.

\section{ACKNOWLEDGMENTS}

We thank M. Keerl for sample preparation. This work has been supported by the Foundation for Fundamental research on Matter (FOM), which is financially supported by the Netherlands Organization for Scientific Research (NWO).
[1] E. R. Weeks, J. C. Crocker, A. C. Levitt, A. Schofield, and D. A. Weitz, Science 287, 627 (2000).

[2] R. E. Courtland and E. R. Weeks, J. Phys.: Condens. Matter 15, S359 (2003).

[3] E. R. Weeks and D. A. Weitz, Phys. Rev. Lett. 89, 095704 (2002).

[4] E. H. Purnomo, D. van den Ende, J. Mellema, and F. Mugele, EPL 76, 74 (2006).

[5] E. H. Purnomo, D. van den Ende, J. Mellema, and F. Mugele, Phys. Rev. E 76, 021404 (2007).

[6] E. H. Purnomo, D. van den Ende, S. A. Vanapalli, and F. Mugele, Phys. Rev. Lett. 101, 238301 (2008).

[7] Y. Gao and M. L. Kilfoil, Phys. Rev. Lett. 99, 078301 (2007).

[8] L. Cipelletti and L. Ramos, J. Phys.: Condens. Matter 17,
R253 (2005).

[9] F. Sciortino and P. Tartaglia, Adv. Phys. 54, 471 (2005).

[10] W. van Megen and S. M. Underwood, Phys. Rev. Lett. 70, 2766 (1993).

[11] J. J. Crassous, M. Siebenbürger, M. Ballauff, M. Drechsler, O. Henrich, and M. Fuchs, J. Chem. Phys. 125, 204906 (2006).

[12] K. Miyazaki, H. M. Wyss, D. A. Weitz, and D. R. Reichman, EPL 75, 915 (2006).

[13] J. M. Brader, Th. Voigtmann, M. E. Cates, and M. Fuchs, Phys. Rev. Lett. 98, 058301 (2007).

[14] P. Sollich, F. Lequeux, P. Hebraud, and M. E. Cates, Phys. Rev. Lett. 78, 2020 (1997).

[15] P. Sollich, Phys. Rev. E 58, 738 (1998).

[16] S. M. Fielding, P. Sollich, and M. E. Cates, J. Rheol. 44, 323 
(2000).

[17] T. G. Mason and D. A. Weitz, Phys. Rev. Lett. 75, 2770 (1995).

[18] W. van Megen, T. C. Mortensen, S. R. Williams, and J. Muller, Phys. Rev. E 58, 6073 (1998).

[19] W. K. Kegel and A. van Blaaderen, Science 287, 290 (2000).

[20] W. van Megen, J. Phys.: Condens. Matter 14, 7699 (2002).

[21] W. van Megen and G. Bryant, Phys. Rev. E 76, 021402 (2007).

[22] W. M. Holmes, P. T. Callaghan, D. Vlassopoulos, and J. Roovers, J. Rheol. 48, 1085 (2004).

[23] M. Cloitre, R. Borrega, and L. Leibler, Phys. Rev. Lett. 85, 4819 (2000).

[24] I. Berndt, J. S. Pedersen, P. Lindner, and W. Richtering, Langmuir 22, 459 (2006).

[25] I. Berndt, J. S. Pedersen, and W. Richtering, J. Am. Chem. Soc. 127, 9372 (2005).

[26] I. Berndt and W. Richtering, Macromolecules 36, 8780 (2003).

[27] T. G. Mason, Rheol. Acta 39, 371 (2000).

[28] T. G. Mason, H. Gang, and D. A. Weitz, J. Mol. Struct. 383,
81 (1996).

[29] J. C. Crocker and D. G. Grier, J. Colloid Interface Sci. 179, 298 (1996).

[30] A. N. Rissanou, D. Vlassopoulos, and I. A. Bitsanis, Phys. Rev. E 71, 011402 (2005).

[31] B. Vorselaars, A. V. Lyulin, K. Karatasos, and M. A. J. Michels, Phys. Rev. E 75, 011504 (2007).

[32] B. Schnurr, F. Gittes, F. C. MacKintosh, and C. F. Schmidt, Macromolecules 30, 7781 (1997).

[33] B. Abou, F. Gallet, P. Monceau, and N. Pottier, Physica A 387, 3410 (2008).

[34] M. Stieger, W. Richtering, J. S. Pedersen, and P. Lindner, J. Chem. Phys. 120, 6197 (2004).

[35] T. Eckert and W. Richtering, J. Chem. Phys. 129, 124902 (2008).

[36] See supplementary material at http://link.aps.org/supplemental/ 10.1103/PhysRevE.81.011404 for the calculation of the pressure, velocity, and stress fields around the tracer particle.

[37] H. Lamb, Hydrodynamics (Cambridge University Press, Cambridge, England, 1975). 